\title{
Non-destructive testing of a rotating glass-fibre-reinforced polymer disc by swept source optical coherence tomography
}

\author{
Jonas Golde ${ }^{1}$, Christian Schnabel ${ }^{1}$, Angelos Filippatos ${ }^{2}$, Tino Wollmann ${ }^{2}$, Maik Gude $^{2}$, Edmund Koch ${ }^{1}$ \\ ${ }^{1}$ Clinical Sensoring and Monitoring, Anesthesiology and Intensive Care Medicine, TU Dresden, 01307 Dresden, Germany \\ ${ }^{2}$ Institute of Lightweight Engineering and Polymer Technology (ILK), TU Dresden, 01307 Dresden, Germany
}

\begin{abstract}
Composite materials are used for high-performance rotating blades, e.g. in turbines and wind power plants. Here, optical coherence tomography was used to visualize large areas of fibre-reinforcedpolymer discs at rotation speeds of up to $1200 \mathrm{rpm}$. These measurements allowed to visualize the fibre structure over large areas of the disc. By recording the front and back reflex, the wobble of the disc was measured precisely. Additionally, the recorded structure was used to detect even small deviations from a uniform rotation.
\end{abstract}

\section{Introduction}

Composite materials are of increasing interest due to a rising demand in efficiency and reliability of fast-rotating blades as used in turbines, power plants and pumps. The tunable successive damage behaviour of multi-layer materials is especially advantageous for the design of rotor blades with a successive and failure-tolerant characteristic. A detailed knowledge about damage formation and structural disorders is necessary to optimize component's life cycle and to achieve costefficient and secure turbine operation. Because vibration characteristics change with different and increasing structural damage, the beginning of those changes needs to be visualized.

Optical Coherence tomography (OCT) is a highresolution three-dimensional imaging modality, suitable to investigate the structure of glass-fibre-reinforced polymer (GFRP) composites as shown previously [1, 2]. Stifter et al. [3] were able to measure the evolving internal stress distribution with polarization-sensitive OCT (PSOCT). In this and future studies, we want to visualize the structure and structural damage of a GFRP disc under dynamic strain caused by fast rotation.

\section{Experimental setup}

A disc of GFRP with a diameter of $200 \mathrm{~mm}$ and nominal $4 \mathrm{~mm}$ thickness was rotated by a stepper motor with velocities ranging from 120 to $1200 \mathrm{rpm}$. The motor controller consisted of an Arduino, generating a stable frequency signal and the ramps for acceleration and deceleration, and a TMC 2208 stepper controller. The beam of a self-developed swept source OCT system (SSOCT), similar to [4], with a centre wavelength of $1300 \mathrm{~nm}$ and an A-scan rate of $200 \mathrm{kHz}$ was focused on the outer part of the rotating disc. The OCT system has an axial measurement range of $8 \mathrm{~mm}$ and an axial resolution of
$16 \mu \mathrm{m}$ in air. This corresponds to a range of $5.17 \mathrm{~mm}$ and a resolution of approximately $10 \mu \mathrm{m}$ in GFRP assuming a refractive index of 1.547. The optical head, consisting of a fibre collimator (F260APC-C, $\mathrm{f}=15.52 \mathrm{~mm}$ ), a deflecting mirror and a focusing lens (AC127-025-C-ML, $\mathrm{f}=25 \mathrm{~mm}$ ), was placed on a linear stage and could be positioned to image the outer $27.5 \mathrm{~mm}$ of the disc. The resulting lateral resolution was approximately $15 \mu \mathrm{m}$; thus, the linear stage was driven with a step size of $10 \mu \mathrm{m}$. The OCT system enabled data acquisition at full speed for extended periods of time. A photo sensor in conjunction with a wire glued to the disc gave a regular signal for every rotation, which was used to trigger the OCT system (B-scan trigger) or recorded as a marker during continuous acquisition. For 3D datasets, the position of the linear stage was changed after each rotation. Due to the stable trigger, the data sets could be sliced in radial direction to form radial cross-sections or combined to enface images.

\section{Results}

High quality OCT images of the fibre structure inside the GFRP could be acquired while the disc was rotating at speeds from 120 to $1200 \mathrm{rpm}$ (Figure 1). Higher velocities were not tested due to safety reasons and will be performed in further studies. From these images, the front and back surface signals were extracted (Figure 2), allowing to precisely measure the wobble of the disc. Moreover, it was found that the thickness of the disc varied between 4.0 and $4.3 \mathrm{~mm}$ (Figure 2 bottom), which was later confirmed by a mechanical measurement. The frequency spectrum (not shown) revealed strongly decreasing amplitudes of the harmonics of the rotational frequency. The highest peak in the frequency spectrum not being a harmonic was at $78 \mathrm{~Hz}$ with an amplitude of $\sim 0.5 \mu \mathrm{m}$, which could be a resonance frequency of the disc. The noise level was well below $10 \mathrm{~nm}$.

* Corresponding author: Edmund.Koch@tu-Dresden.de 
Furthermore, the fingerprint-like unique pattern of the fibres inside the GFRP allowed detecting even faint deviations from a uniform revolution. While the pattern could be matched to a single A-scan $(5 \mu \mathrm{s})$, the noise level of a periodic deviation was less than $0.1 \mu \mathrm{s}$, corresponding to $10^{-6}$ of a revolution (Figure 3 ).
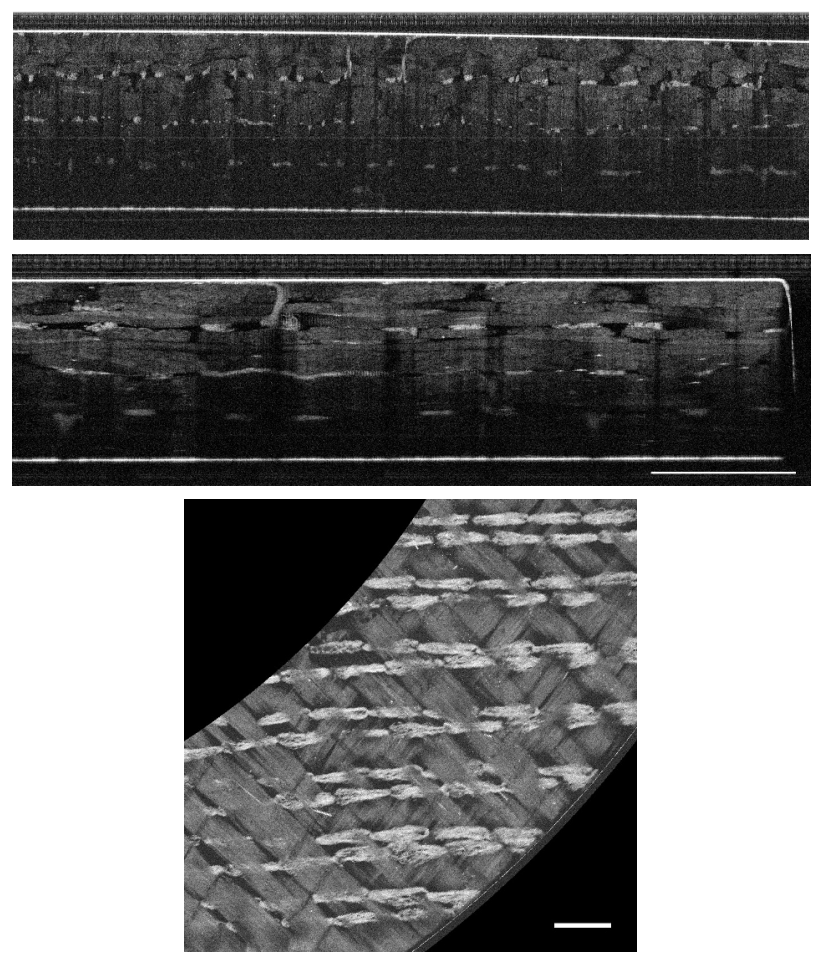

Fig. 1. OCT images of the rotating disc captured at $1200 \mathrm{rpm}$. Top: Azimuthal B-scan, the width corresponds to a length of $115 \mathrm{~mm}$ while the height is only $8 \mathrm{~mm}$ in air. Middle: Cross section in radial direction. Bottom: enface image at approx. $1.3 \mathrm{~mm}$ depth within the disc. Scale bars are $5 \mathrm{~mm}$ in air.

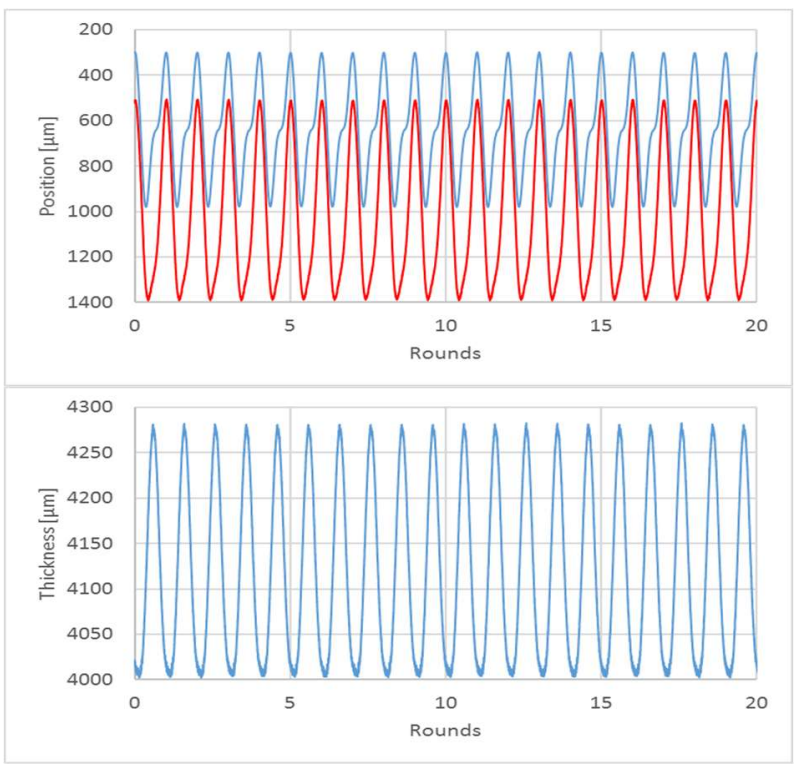

Fig. 2. Top: Signal of the front (blue) and back (red) surface of the disc over 20 rotations, measured from one side of the disc. The signal of the back surface (lower trace) was shifted to be visible on the same scale. Bottom: Thickness of the disc calculated from the signals of both surfaces.

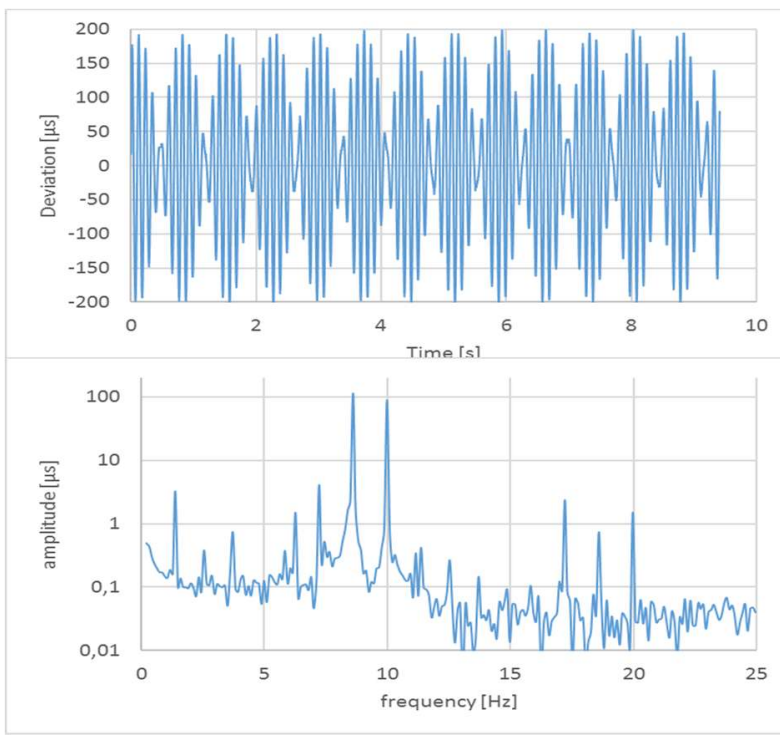

Fig. 3. Top: Deviations of the angular position of the disc from a uniform rotation as indicated by the unique pattern of the fibres inside the GFRP measured while rotating at $600 \mathrm{rpm}$. Bottom: Corresponding frequency spectrum showing peaks at $10 \mathrm{~Hz}$ (rotation frequency) and $8.6 \mathrm{~Hz}$ with similar amplitude. Other frequencies (harmonics and mixing frequencies) have amplitudes, which are at least one order of magnitude smaller. The noise level, visible at frequencies above $12 \mathrm{~Hz}$, is below $0.1 \mu \mathrm{s}$. The frequency of $8.6 \mathrm{~Hz}$ seems to be a consequence of the magnetic forces given by the motor and the moment of inertia of the disc.

\section{Conclusions}

Swept source OCT can be used to measure the structure of GFRP while rotating at fast speed. The surface position as well as the (optical) thickness of the disc could be measured with high accuracy. The structure of the GFRP allowed identifying the position with a resolution of $5 \mu \mathrm{s}$, corresponding to $0.018^{\circ}$ at $600 \mathrm{rpm}$. While radial acceleration at this velocity is more than $150 \mathrm{~g}$, this does not endanger the structure of the disc. Measurements at discs rotating 5 to 10 times faster, possible with faster OCT systems, could show the degradation of the material and lead to a better understanding of the dynamics during material failure.

\section{References}

1. P. Liu, Roger M. Groves, R. Benedictus. Ndt \& E International 64: 52-58 (2014)

2. Joy P Dunkers, et al., Composites Part A: Applied Science and Manufacturing 30.2: 139-145 (1999).

3. D. Stifter, E. Leiss-Holzinger, Z. Major, B. Baumann, M. Pircher, E. Götzinger, C. K. Hitzenberger, B. Heise, Opt. Express 18, 25712-25725 (2010).

4. L. Kirsten, M. Schindler; J. Morgenstern; M. T. Erkkilä; J. Golde; J. Walther; P. Rottmann; M. Kemper; M. Bornitz; M. Neudert; T. Zahnert; E. Koch, Journal of biomedical optics 24.3: 031017 (2018). 